\title{
On the Generation of Oriented Matroids*
}

\author{
J. Bokowski ${ }^{1}$ and A. Guedes de Oliveira ${ }^{2}$ \\ ${ }^{1}$ Mathematics Department, Darmstadt University of Technology, \\ Schloßgartenstrasse 7, D-64289 Darmstadt, Germany \\ bokowski@mathematik.tu-darmstadt.de \\ ${ }^{2}$ Pure Mathematics Department, University of Porto, \\ Praça Gomes Teixeira, P-4050 Porto, Portugal \\ agoliv@fc.up.pt
}

\begin{abstract}
We provide a multiple purpose algorithm for generating oriented matroids. An application disproves a conjecture of Grünbaum that every closed triangulated orientable 2-manifold can be embedded geometrically in $\mathbb{R}^{3}$, i.e., with flat triangles and without selfintersections. We can show in particular that there exists an infinite class of orientable triangulated closed 2-manifolds for each genus $g \geq 6$ that cannot be embedded geometrically in Euclidean 3-space. Our algorithm is interesting in its own right as a tool for many investigations in which oriented matroids play a key role.
\end{abstract}

\section{Introduction}

The inductive generation of oriented matroids or chirotopes is possible in many ways, reflecting the variety of possible characterizations of these topological invariants. In particular, this has been used in connection with geometrical embeddability problems, see, e.g., [9], [7], [8], and [1]-[3]. Compared with the generation methods used in those articles, we present a much more efficient algorithm. Its application proved for the first time that there exists a nonembeddable orientable triangulated closed 2-manifold of genus 6. Moreover, we obtain the result that there exists an infinite class of orientable triangulated closed 2-manifolds for each genus $g \geq 6$ that cannot be embedded geometrically in Euclidean 3-space.

When is a given finite poset isomorphic to the face poset of some polyhedral complex in a given space $\mathbb{R}^{n}$ ? In the two-dimensional case we have, on the one hand, Steinitz's

\footnotetext{
* The research of the second author was partially supported by FCT and PRAXIS XXI through the Research Units Pluriannual Funding Program, and by the project AGC/PRAXIS XXI/2/2.1/MAT/63/94.
} 
solution for face posets of spherical maps [24] as a complete answer in the genus $g=0$ case, but, on the other hand, "very little is known about polyhedral embeddings of orientable polyhedral maps of positive genus $g$. For example, for each $g \geq 1$ it is an open question whether or not each simplicial polyhedral map on the orientable closed surface of genus $g$ can be polyhedrally embedded in 3-space $\mathbb{R}^{3}$," see [11].

It was conjectured by Grünbaum that every closed orientable triangulated 2-manifold without boundary can be embedded geometrically in three-dimensional Euclidean space $\mathbb{R}^{3}$, see [15] and Chapter 13.2, Exercise 3, of [13]. In the meantime many mathematicians expected the answer to this problem to be negative for sufficiently large genus $g$ of the 2-manifold, but they could not prove it. For an interesting attempt using cohomology groups see [19].

In Section 2 we describe an example from Altshuler's list of neighborly 2-manifolds of genus 6 which cannot be embedded geometrically in $\mathbb{R}^{3}$. The nonembeddability result depends on our algorithm for generating oriented matroids which is of interest in its own right. We present our algorithm in Section 3.

Our key example for the embeddability problem can be seen in connection with the Heawood conjecture about the map coloring of surfaces of genus $g$. The lower bound for the number of colors which are sufficient for map coloring on a surface of genus $g$ is the greatest natural number $\chi(g)$ smaller than or equal to $\frac{1}{2}(7+\sqrt{48 g+1})$. The fact that this bound is the best possible (Heawood conjecture) was proven with two exceptions by Ringel, Youngs (see [22]), and others, and is summarized by Ringel in [21]. The exceptions are the Klein bottle and the sphere (or plane). For the latter four color problem, see [23] and the papers cited there.

The first equality case in the Heawood inequality corresponds to the tetrahedron. The second one leads to Möbius' torus [18], with its polyhedral Császár realization, see [12]. In the genus $g=6$ case, the third equality case, $\chi(g)=\frac{1}{2}(7+\sqrt{48 g+1})$, we can embed the complete graph with 12 vertices without self-intersections on a closed orientable 2-manifold with genus 6 in many nonisomorphic ways. The dual map on this surface is the relevant one for map coloring.

Among the candidates of closed orientable triangulated 2-manifolds of genus 6 with 12 points, we have the oldest example of Hefter from 1891, see [16] or [20]. We find another example by Ringel in [20]. The examples of Hefter and Ringel formed the starting point for Altshuler's investigation to determine 59 neighborly 2-manifolds with 12 vertices. The first author showed that Altshuler's list is complete. For this Altshuler list see [3].

The geometrical embeddability could not be decided in any of these 59 cases in [3], except under symmetry assumptions. Here we present the embeddability decision in Case 54 from [3].

\section{Altshuler's $K_{12}$-Map No. 54}

The neighborly 2-manifold with 12 vertices from Altshuler's list, map No. 54 in [3], is of genus 6 and it has a symmetry group $G$ of order 6 . The symmetry group acts on the set of vertices in such a way that we have two orbits with six elements each. The symmetry suggests a relabeling of the vertices compared with [3]. We have chosen the following 
permutation of the vertices:

$\begin{array}{ll}\text { old labels } & 1,2,3,4,5,0,7,8,9,6, \mathrm{a}, \mathrm{b} \\ \text { final labels } & 12,11,10,9,8,7,6,5,4,3,2,1\end{array}$

The two orbits under the symmetry group $G$ in the set of vertices are $E_{1}=\{1,2,3,4$, $5,6\}$ and $E_{2}=\{7,8,9,10,11,12\}$. The abstract 2-manifold is given by the following 44 triangles:

$\begin{aligned} & |1,2,5| 1,2,12|1,3,4| 1,3,5|1,4,11| 1,6,7|1,6,9| 1,7,8|1,8,10| 1,9,12|1,10,11| \\ & |2,3,10| 2,3,11|2,4,6| 2,4,7|2,5,6| 3,4,6|3,5,9| 3,6,8|2,7,10| 2,8,9|2,8,12| \\ & |2,9,11| 3,7,9|3,7,12| 3,8,11|3,10,12| 4,5,8|4,5,12| 4,7,9|4,8,11| 4,9,10|4,10,12|\end{aligned} \mid$

The generators of the symmetry group $G$ are

$$
(7,12,11)(10,9,8)(3,6,5)(4,2,1) \text { and }(7,10)(12,8)(11,9)(3,1)(6,2)(5,4) \text {. }
$$

With the algorithm of Section 3 we proved that no oriented matroid on 12 points (not even a nonrealizable one) is admissible with Altshuler's 54 map. In particular, no polyhedral realization of the map is possible. We first constructed all the six-element oriented matroids admissible with the restriction of the map to the vertices $1, \ldots, 6$ and then iteratively computed all the admissible extensions to the other six points. The symmetry group $G$ of the map helped us in that the 724 oriented matroids which are admissible with the first six vertices fall into 143 orbits under the action of $G$ and we only needed to start the process with one representative of each orbit. This was the reason for choosing this particular map No. 54. Although a relatively big number of oriented matroids with 11 elements occur, no one with 12 points exists. We have therefore the following theorem:

Theorem 2.1. Not every closed triangulated orientable 2-manifold can be embedded in $\mathbb{R}^{3}$ with flat triangles and without self-intersections. More precisely, the 54th map in Altshuler's list cannot be embedded geometrically in $\mathbb{R}^{3}$. In fact, there is no matroid manifold for this map, an oriented matroid with 12 points both acyclic and compatible with it.

However, such an abstract 2-manifold can always be realized topologically. In our case we can even find a topological embedding of the 2-manifold in which the symmetry group $G$ is induced by a subgroup of the group of proper rigid motions, see Fig. 1.

\section{The Algorithm}

\subsection{Hyperline Configurations}

Among the many concepts to work with oriented matroids, we have the list of hyperline configurations which was used by the first author for the first time in 1978. We refer the reader to Chapter VII of [10], or to [6]. Here we need in particular the uniform case in rank 4 in which the hyperlines are lines. The concept is closely related to the clusters of stars of Goodman and Pollack, see [14]. 


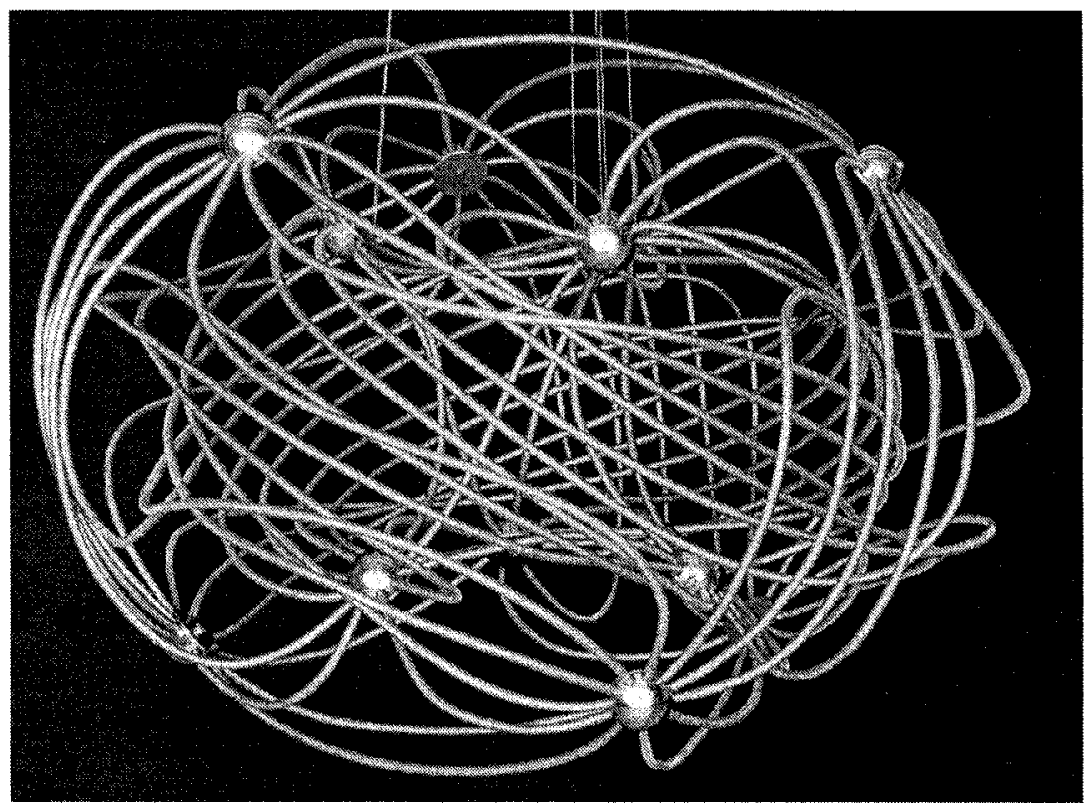

Fig. 1. Model of the complete graph with 12 vertices in 3-space which also shows the circular order of all topological triangles around each point. These triangles form the above neighborly 2-manifold of Altshuler. This corresponding topological triangulated 2-manifold of genus 6 has a Euclidean symmetry of order 6 . Photograph of N. Hähn of a model made by the first author, colored version: www.juergen.bokowski.de.

The realizable case can serve to introduce the hyperline configurations. We consider an ordered set $E=\left\{x_{1}, x_{2}, \ldots, x_{n}\right\}, n \geq 4$, of affine points in general position in Euclidean 3 -space $\mathbb{R}^{3}$. We fix an orientation in $\mathbb{R}^{3}$ by defining one of the two equivalence classes of affine bases in $\mathbb{R}^{3}$ to be the positive one, e.g., we require $\left(x_{1}, x_{2}, x_{3}, x_{4}\right)$ to be a positive basis. The normal vector at $x_{3}$ of the plane spanned by $x_{1}, x_{2}, x_{3}$ which points into the half-space containing $x_{4}$ defines a positive rotation around the oriented line defined by $\left(x_{1}, x_{2}\right)$. For any ordered pair $\left(x_{i}, x_{j}\right), i<j$, of points in $E$ we have a corresponding oriented line $L_{i, j}$, and we define similarly a positive rotation around the oriented line.

Each 3-tuple $\left(x_{i}, x_{j}, x_{k}\right), x_{k} \in E \backslash\left\{x_{i}, x_{j}\right\}$ of points forms an affine basis of a corresponding oriented plane $E_{i, j}^{k}$ spanned by these points. For each oriented line $L_{i, j}$, the corresponding unoriented planes $|E|_{i, j}^{k}$ define a periodical infinite sequence

$$
L_{i, j}: \quad \ldots,|E|_{i, j}^{k_{1}},|E|_{i, j}^{k_{2}}, \ldots,|E|_{i, j}^{k_{n-2}}, \ldots
$$

with period length $n-2$.

We associate to the line $L_{i, j}$ a list $\left(k_{1}, s_{2} \cdot k_{2}, \ldots, s_{n-2} \cdot k_{n-2}\right)$ of signed indices, where $\left(k_{1}, \ldots, k_{n-2}\right)$ is the list of indices of points in $E \backslash\left\{x_{i}, x_{k}\right\}$ starting with the smallest index $k_{1}$ and sorting the others according to the positive rotation around the oriented line $L_{i, j}$, and where $s_{l} \in\{-1,+1\}$ indicates whether the point $x_{l}$ is passed over in the same half-plane as $x_{k_{1}}$ when rotating the plane $\left(x_{i}, x_{j}, x_{k_{1}}\right)$ a half turn in the positive direction around $L_{i, j}$. In other words, $s_{l}$ equals the sign of the determinant of the 4-tuple 
$\left(x_{i}, x_{k}, x_{k_{1}}, x_{l}\right)$. In the same way, the sign of the determinant of $\left(x_{i}, x_{j}, x_{k_{l}}, x_{k_{m}}\right)$ equals $s_{k_{l}} \cdot s_{k_{m}}$ if $l<m$ and the opposite if $l>m$. We denote these determinants by a bracket $\left[i, j, k_{l}, k_{m}\right]$.

Moreover, such a complete list of sequences of signed indices forms a general uniform oriented matroid of rank 4 if and only if each index occurs precisely once in each line and when different lines defining the same bracket sign via the alternating rule for bases do not violate that rule, see, e.g., Proposition 7.2 of [10] or see [5]. We use this as a definition of a (general, uniform) oriented matroid of rank 4 and as a basis for the algorithm we present next. The chirotope is the function (corresponding in the realizable case to the sign of determinant) that assigns to every ordered set of four indices either 1 or -1 , accordingly. As in matroid theory, the terms hyperline or line and hyperplane or plane are used in this general setting, meaning a set of two or three different indices, respectively, and by cocircuit we mean the complement of a hyperplane, i.e., the set of indices of points not in a given hyperplane.

It is an immediate consequence of this definition that we may extend a uniform oriented matroid by one point in general position in all possible ways just by choosing the "gaps" on the line sequences where the new point lies. In fact, all bracket signs are fixed when we consider only the lines prior to the extension. All gaps are possible, provided the former rules are obeyed. Moreover, by doing this, it is possible to prove the signs are already those of a chirotope, meaning that it is also possible to determine the sequences for the lines containing the new point in such a way that the alternating rule is never violated [17]. We make this more precise in the following example.

\subsection{The Algorithm in the Smallest Example}

We start with a hyperline configuration for the four vertices of a 3-simplex. Up to reordering the indices, there is only one bracket to consider, of lowest index, 0 .

\begin{tabular}{|c|c|c|c|c|c|c|c|}
\hline line & & 0 & 1 & column & bracket & index & sign bracket \\
\hline 0 & $(1,2$ & 3 & +4 & ) & & 0 & $+[1,2,3,4]$ \\
\hline 1 & $(1,3$ & 2, & -4 & ) & & & \\
\hline 2 & $(1,4$ & 2, & +3 & ) & & & \\
\hline 3 & $(2,3$ & 1, & +4 & ) & & & \\
\hline 4 & $(2,4$ & 1, & -3 & ) & & & \\
\hline 5 & $(3,4$ & 1, & +2 & ) & & & \\
\hline
\end{tabular}

We find any one element extension as follows, depending on the choices we make. For example, we pick a sign for the new element 5 for inserting it in line 0 (first choice + ). Now we pick a gap position for +5 in line 0 (second choice, gap between column 0 and column 1). The second possibility would have been the gap after column 1 . These two choices imply the following two signs in the chirotope: $\operatorname{sign}[1,2,3,+5]=$ $\operatorname{sign}[1,2,+5,+4]=1$. The alternating rule for determinants implies that $\operatorname{sign}[1,3,2$, $-5]=\operatorname{sign}[1,4,2,+5]=\operatorname{sign}[2,3,1,+5]=\operatorname{sign}[2,4,1,-5]=1$, which tells us that the sign of element 5 when inserted in lines $1,2,3$, and 4 is, respectively, ,++ , and - . In general, for each plane index with known bracket sign there will be 
two (line, column) other positions pertaining to the same plane which either define the sign of the new element in that line or restrict the possible gap positions in that line.

\begin{tabular}{|c|c|c|c|c|c|c|c|c|c|c|c|c|c|}
\hline \multirow{2}{*}{$\frac{\text { line }}{0}$} & \multirow[b]{2}{*}{$(1,2$} & \multirow{2}{*}{$\frac{0}{3,}$} & \multirow{2}{*}{$\begin{array}{r}1 \\
+5,\end{array}$} & \multicolumn{3}{|c|}{ column } & \multirow{2}{*}{$\frac{\text { bracket }}{0}$} & \multirow{2}{*}{ index } & \multicolumn{3}{|c|}{ sign bracket } & \multicolumn{2}{|c|}{ plane index } \\
\hline & & & & +4 & ) & +5 & & & + & {$[1,2,3,4$} & & & \\
\hline 1 & $(1,3$ & 2 , & -4 & ) & & -5 & 1 & & + & {$[1,2,3$,} & 5 & ] & \\
\hline 2 & $(1,4$ & 2, & +3 & ) & & +5 & 2 & & - & {$[1,2,4$,} & 5 & ] & \\
\hline 3 & $(2,3$ & 1, & +4 & ) & & +5 & 3 & & 0 & {$[1,3,4$,} & 5 & ] & \\
\hline 4 & $(2,4$ & 1, & -3 & ) & & -5 & 4 & & 0 & {$[2,3,4$,} & 5 & ] & \\
\hline 5 & $(3,4$ & 1, & +2 & ) & & & & & & & & & \\
\hline
\end{tabular}

Now (third choice) we pick the first gap for the element -5 in line 1 (between 2 and -4 ) which determines the sign for the bracket with bracket index 3 (or plane index 2 ); $\operatorname{sign}[1,3,-5,-4]$ has to be positive. We go to the other two ( 1 ine, column) positions for plane $1,3,4:(5,0)$ and $(2,1)$. $(5,0)$ implies the sign for element 5 when inserted in line 5 has to be negative ( $\operatorname{sign}[3,4,1,-5]=1$ must hold). $(2,1)$ restricts the possible gap positions in line 2 for element +5 : $\operatorname{sign}[1,4,+3,+5]=1$ must hold, i.e., only the second gap position is allowed. We have a last choice for the gap position of element +5 in line 3 . We pick (last choice) the first gap in line 3 which forces $\operatorname{sign}[2,3,+5,+4]=1$. We obtain the following list of hyperline configurations when we fill the remaining lines as defined by the brackets. This implies a sorting argument for the new line 3, the new line and column values have changed, the new planes (cocircuits) relevant for the next one element extensions with its ( line, column)-values can be evaluated. The table contains also the indices of the planes in the (line, column) positions.

\begin{tabular}{|c|c|c|c|c|c|c|c|c|c|c|c|c|c|c|c|}
\hline \multirow[b]{2}{*}{ line } & & \multirow[b]{2}{*}{0} & \multirow[b]{2}{*}{1} & \multirow{2}{*}{\multicolumn{2}{|c|}{2}} & \multicolumn{4}{|c|}{ new planes } & \multirow[b]{2}{*}{ br.i. } & \multirow{2}{*}{\multicolumn{2}{|c|}{ sign bracket }} & \multirow[b]{2}{*}{ p. $-i$} & \multirow[b]{2}{*}{ new $p$. } & \multirow[b]{2}{*}{$i$} \\
\hline & & & & & & 0 & 1 & 2 & & & & & & & \\
\hline 0 & $(1,2$ & 3 & +5 & +4 & ) & 0, & +2, & +1 & ) & 0 & $+[1,2,3,4]$ & & & 123 & 0 \\
\hline 1 & $(1,3$ & 2, & -5 & -4 & ) & 0 & -4 & -3 & ) & 1 & $+[1,2,3$, & $5]$ & 0 & 124 & 1 \\
\hline 2 & $(1,4$ & 2, & +3 & +5 & ) & 1, & +3 & +5 & ) & 2 & $-[1,2,4$, & $5]$ & 1 & 125 & 2 \\
\hline 3 & $(1,5$ & 2, & +3 & -4 & ) & 2, & +5 & -4 & ) & 3 & $-[1,3,4$, & $5]$ & 2 & 134 & 3 \\
\hline 4 & $(2,3$ & 1, & +5 & +4 & ) & 0, & +7 & +6 & ) & 4 & $-[2,3,4$, & $5]$ & 3 & 135 & 4 \\
\hline 5 & $(2,4$ & 1, & -3 & -5 & ) & 1, & -6 & -8 & ) & & & & & 145 & 5 \\
\hline 6 & $(2,5$ & 1, & -3 & +4 & ) & 2, & -7 & +8 & ) & & & & & 234 & 6 \\
\hline 7 & $(3,4$ & 1, & +2 & -5 & ) & 3, & +6 & -9 & ) & & & & & 235 & 7 \\
\hline 8 & $(3,5$ & 1, & +2 & +4 & ) & 4, & +7 & +9 & ) & & & & & 245 & 8 \\
\hline 9 & $(4,5$ & 1, & -3 & -2 & ) & 8, & -9 & -2 & ) & & & & & 345 & 9 \\
\hline
\end{tabular}

We insist that it should be possible to obtain in this way all one point extensions of the former chirotope (the simplex), i.e., all uniform, five elements, rank 4 chirotopes, up to reversing all signs. 


\subsection{A Rank 3 Example}

With the following example we explain the decisive improvement of the algorithm which allowed the calculations in the case of map 54 of Altshuler's list.

We now use only a partial chirotope (just the part corresponding to lines containing point 1). In oriented matroid language, this is the contraction at element 1 , and it is a rank 3 oriented matroid:

\begin{tabular}{ll|rrrrrrr}
$((1)$, & 2 & 3 & 7 & 8 & 6 & 5 & 4 & ) \\
$((1)$, & 3 & 2 & -7 & -6 & -5 & -8 & -4 & ) \\
$((1)$, & 4 & 2 & 3 & 7 & 8 & 6 & 5 & ) \\
$((1)$, & 5 & 2 & 8 & 3 & 7 & 6 & -4 & ) \\
$((1)$, & 6 & 2 & 8 & 3 & 7 & -5 & -4 & ) \\
$((1)$, & 7 & 2 & 3 & -6 & -5 & -8 & -4 & )
\end{tabular}

We delete point 8 in the rank 3 contraction of point 1 and we look at the method for finding this one element extension. We pick the interval between the hyperplanes $((1)) 2,7$,$) and ((1)) 2,6$,$) and we pick an orientation of the pseudoplane 8$. This defines the hyperline

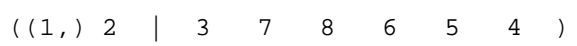

It also defines the sign of 8 in all other hyperlines as follows since the corresponding signs of brackets have been determined:

\begin{tabular}{ll|rrrr}
$((1)$, & 3 & 2 & $\ldots$ & -8 & ) \\
$((1)$, & 4 & 2 & $\cdots$ & 8 & ) \\
$((1)$, & 5 & 2 & $\cdots$ & 8 & ) \\
$((1)$, & 6 & 2 & $\cdots$ & 8 & ) \\
$((1)$, & 7 & 2 & $\ldots$ & -8 & )
\end{tabular}

Now we pick an open interval (gap) between two cocircuits on hyperline 3 (as in the example):

$$
\left((1,) \quad 3 \quad \mid \begin{array}{lllllll}
2 & 2 & -7 & -6 & -5 & -8 & -4
\end{array}\right)
$$

The corresponding brackets $[(1) 3, x, 8$,$] for all elements x$ are now determined. This reduces the possible gap positions in the remaining lines. The former gap bounds are changed as indicated by the arrows. Now it is decisive not to insert the new element immediately in one of the remaining gaps. We can see from hyperline $((1) 5$,$) and$ $((1) 6$,$) (the gap bound has jumped over several hyperplanes) that additional signs of$ brackets are already determined, even before reaching these lines. This is the key observation for the new algorithm. For example, we do know already, as a consequence of our choice in line $(1)$,3 , that the sign of $[(1) 5,8,-4$,$] is +1$. We may set this sign at this early stage, and even evaluate the consequences, thence shortcutting a number of trials ahead. This leads to restricted intervals for the gaps and this again can lead to additional signs of brackets, etc. Note that, had we not used the gap movement, this sign would only be fixed while trying to set the signs for line $((1) 5$,$) , i.e.,$ while running process_line for line 4 . This is the crucial improvement from earlier algorithms. 
Again, this can be summarized as follows:

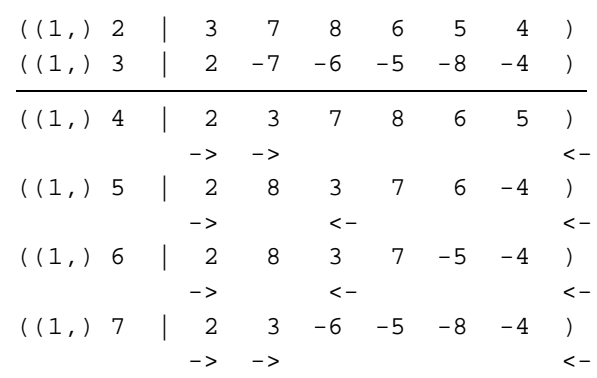

\subsection{The Algorithm}

1. We pick a sign for the new element in line 0 .

2. Each bracket corresponding to a plane index is set to zero.

3. For each line we define that the left gap has to lie after column 0 .

4. For each line we define that the right gap has to lie before the last column +1 .

5. We run the following procedure for a line equal to 0 :

6. Procedure process_line for line:

6.1. If left gap is greater than right gap or line is greater than last_line then return.

6.2. Pick the next gap in line according to the gap conditions, and increase by one the left gap.

6.3. For column from 0 to $n-2$ run the following procedure:

6.4. Procedure set_sign for ( 1 ine, column) :

6.4.1. Pick the new sign in line relative to plane in (line, column) and evaluate the reduced gap intervals in the lines ahead pertaining to the same plane. If in some line the gap jumps over any hyperplane, run set_sign for the ( 1 ine, column) where it occurs. If there is no empty gap, set these new gap intervals and run procedure process_line for line $=$ next line; otherwise return.

\subsection{Matroid Manifold}

When studying embeddability of a simplicial 2-map, we do not want all possible oriented matroids, but only those which are admissible with the map in the following two ways:

First, any oriented matroid realized by affine points is acyclic, meaning that every element belongs to a positive cocircuit or, equivalently, that there is no positive circuit.

Second, any polyhedral embedding of the map in $\mathbf{R}^{3}$ satisfies that any disjoint triangle $[i, j, k]$ and edge $[l, m]$ of the map do not intersect each other in the embedding. This 
is equivalent to saying that we do not have $\operatorname{sign}[i, j, k,-l]=-\operatorname{sign}[i, j, k,-m]=$ $\operatorname{sign}[i, j,-l,-m]=-\operatorname{sign}[i, k,-l,-m]=\operatorname{sign}[j, k,-l,-m]$ or, in oriented matroid terms, that $(i, j, k,-l,-m)$ is not a positive circuit.

We say that an oriented matroid is admissible with the map if it is acyclic and it satisfies the above requirement for every pair of triangle and edge in the map. A polyhedral map together with an oriented matroid admissible with it in this sense was called a matroid manifold in [5] and [10].

The verification of acyclicity and compatibility can be done automatically, and the corresponding checking procedures can be implemented together with the algorithm, as a (multiple) test in process_line. We do this so that they determine new signs whenever possible, instead of discarding their opposites afterward.

We now consider the final algorithm that extends successively the points and checks for acyclicity and compatibility with a given map. In order to output the chirotopes before evaluating the subsequent ones, to store at any stage as less information as possible, after outputting a chirotope we proceed by changing the last choice, and when we run out of possibilities we go back to the previous choice and so on. This can be done by replacing process_line in the the algorithm already presented (starting with $n=4$ and the corresponding cocircuit sequences already built) by the following:

6. (New) procedure process_line for line:

6.1. If left gap is not greater than right gap or line is not greater than last_line go to 6.2. If $n$ is equal to the last_element return. Otherwise, build the cocircuit sequences for the new chirotope and go to 1 .

6.2. $\cdots$

6.3. $\cdots$

6.4. (New) procedure set_sign for (line, column):

6.4.1. Pick the new sign in line relative to plane in (line, column) and evaluate the reduced gap intervals in the lines ahead pertaining to the same plane. Check whether for any positive or forbidden circuit four signs are fixed as predicted. If so, evaluate all (column,line) pairs where it occurs and run set_sign for them. If in some line the gap jumps over any hyperplane, run also set_sign for the (line, column) where it occurs. If there is no empty gap, set these new gap intervals and run procedure process_line for line $=$ next line; otherwise return.

\subsection{Remarks about the Algorithm}

(a) The algorithm also works in the nonuniform case and in arbitrary rank. Of course, the implementation needs much more time.

(b) The generation of reorientation classes only can easily be done by always picking the positive sign in line 0 for the new element.

(c) In the rank 3 case one can show that filling the possible gaps (after the restricted intervals have been determined) always leads to an oriented matroid. In this respect, the algorithm is as efficient as possible in the rank 3 case. 


\section{Implementation of the Algorithm and Further Results}

This algorithm was implemented by the first author, using "C++," and, independently, by the second author using " $\mathrm{C}$," in rather different programs leading to the same results wherever used. These programs can be obtained via e-mail from either of the authors.

This problem has long been a challenging one among what has been called computational synthetic geometry, and many articles can now be seen as attempts to reach this final goal.

The basis for our algorithm (without using "second-order consequences," the information in 6.4.1 about the reducing of the gaps) was implemented before on various occasions: A first implementation, Auffüll (Anheuser and Bokowski), was done in Fortran 77, more than 12 years ago. More recently, a second implementation in Pascal, radonchi (Schuchert), was written using the circuits axioms of oriented matroids. The performance was similar to the foregoing version. Comparing both implementations was possible and both versions always lead to equal results. Moreover, the realizations of polyhedral structures always supported the correctness of these early implementations.

Another implementation in the language C, fill_in, was written by Guedes de Oliveira in 1992. Again the main motivation was to obtain a faster implementation. Unfortunately, this version had about the same performance. However, again, testing one program against the other was possible and this was a very good aspect for confirming all computations.

The next implementation of the first author together with Biermann was written in a functional language, gofer (good for equational reasoning). Gofer is a part of Haskell and freely available. This implementation finally used also the second-order consequences idea of the first author. The performance is rather bad but the language is considered to be a reliable one. Again all tests with former programs confirmed the results.

It could have happened that there existed an oriented matroid with these properties, but yet not realizable. This was not the case. There is no corresponding matroid manifold. Moreover, we deleted triangle $(7,11,12)$ from Altshuler's $K_{12}$-map No. 54 and we started all computations once more for the corresponding 2-manifold with boundary. This time the symmetry was only a threefold one and the computations took longer than before. (We cannot tell precisely the amount of CPU-time we used altogether but it far exceeded the 10 years limit.) The result was again that there does not exist a corresponding matroid manifold. This did not only confirm our former result but it lead to the following theorem.

Theorem 4.1. There are infinite classes of closed triangulated orientable 2-manifolds for each genus $g \geq 6$ which cannot be embedded in $\mathbb{R}^{3}$ with flat triangles and without self intersections.

The reason for that is simple since we can glue to our 2-manifold instead of the triangle $(7,11,12)$ any triangulated closed 2-manifold in which one triangle was removed. 


\section{Acknowledgments}

Many people supported us in getting additional CPU-time for our processes. We thank for that Boris Bokowski from Berlin, Ilda da Silva from Lisbon, Gerhard Wesp and Johann Linhart from Salzburg, Francisco Sepúlveda Teixeira and the Mathematics Department of I.S.T. from Lisbon, Heidi Burgiel from Minnesota, Peter Gritzmann from München, Ileana Streinu from Smith College, Massachusetts. We wish to thank in addition Boris Bokowski for all his advice in getting the computations done on various computers. Finally, we thank the referee for his valuable suggestions and various improvements.

\section{References}

1. Altshuler, A., Bokowski, J., Schuchert, P., Spatial polyhedra without diagonals, Israel J. Math., 86 (1994), 373-396.

2. Altshuler, A., Bokowski, J., Schuchert, P., Sphere systems and neighborly spatial polyhedra with 10 vertices, Suppl. Rend. Circ. Mat. Palermo (2), 35 (1994), 15-28.

3. Altshuler, A., Bokowski, J., Schuchert, P., Neighborly 2-manifolds with 12 vertices, J. Combin. Theory Ser. A, 75(1) (1996), 148-162.

4. Björner, A., Las Vergnas, M., Sturmfels, B., White, N., Ziegler, G. M., Oriented Matroids, Encyclopedia of Mathematics and its Applications, Vol. 46, Cambridge University Press, Cambridge, 1993.

5. Bokowski, J., On the geometric flat embedding of abstract complexes with symmetries, in: Symmetry of Discrete Mathematical Structures and Their Symmetry Groups. A Collection of Essays, pp. 1-48, Hofmann, K. H. and Wille, R. (eds.), Research and Exposition in Mathematics, Vol. 15, Heldermann, Berlin, 1991.

6. Bokowski, J., Oriented matroids, in: Handbook of Convex Geometry, Gruber, P., and Wills, J. M. (eds.), Elsevier, North-Holland, Amsterdam, 1992.

7. Bokowski, J., Brehm, U., A New Polyhedron of Genus 3 with 10 Vertices, Colloquia Mathematica Societate János Bolyai, North-Holland, Amsterdam, 1985.

8. Bokowski, J., Brehm, U., A polyhedron of genus 4 with minimal number of vertices and maximal symmetry, Geom. Dedicata, 29 (1989), 53-64.

9. Bokowski, J., Eggert, A., All realizations of Möbius' torus with seven vertices, Structural Topology, 20 (1991), 59-78.

10. Bokowski, J., Sturmfels, B., Computational Synthetic Geometry, Lecture Notes in Mathematics, Vol. 1355, Springer-Verlag, Berlin, 1989.

11. Brehm, U., Schulte, E., Polyhedral maps, in: Handbook of Discrete and Computational Geometry, pp. 345358, Goodman, J. E., and O’Rourke, J. (eds.), CRC Press, Boca Raton, FL, 1997.

12. Császár, A., A polyhedron without diagonals, Acta. Sci. Math. (Szeged), 13 (1949), 140-142.

13. Duke, R. A., Geometric embedding of complexes, Amer. Math. Monthly, 77 (1970), 597-603.

14. Goodman, J. E.; Pollack, R.: Semispaces of configurations, cell complexes of arrangements, J. Combin. Theory Ser. A, 37(3) (1984), 257-293.

15. Grünbaum, B.: Convex Polytopes, Pure and Applied Mathematics, Vol. 16, Interscience-Wiley, New York, 1967.

16. Hefter, L., Über das Problem der Nachbargebiete, Math. Ann., 38 (1891), 477-508.

17. Las Vergnas, M.: Extensions ponctuelles d'une géométrie combinatoire orienté, in: Problèmes combinatoires et théorie des graphes (Actes Coll., Orsay, 1976), Colloques internationaux, C.N.R.S., No. 260, pp. 265-270, 1976.

18. Möbius, A. F., Gesammelte Werke II, Hrsg. Felix Klein, Neudruck der Ausgabe von 1886, (1967), pp. $552 \mathrm{ff}$.

19. Novik, I., Note on geometric embeddings of simplicial complexes in a Euclidean space, Private communication.

20. Ringel, G., Färbungsprobleme auf Flächen und Graphen, VEB Deutscher Verlag der Wissenschaften, Berlin, 1959. 
21. Ringel, G., Map Colour Problem, Springer-Verlag, Berlin, 1974.

22. Ringel, G., Youngs, J. W. T., Solution of the Heawood map-coloring problem, Proc. Nat. Acad. Sci. USA, 60 (1968), 438-445.

23. Robertson, N., Sanders, D., Seymour, P., Thomas, R., The four-colour theorem, J. Combin. Theory Ser. B, 70 (1997), 2-44.

24. Steinitz, E., Polyeder und Raumeinteilungen, Enzykl. math. Wiss., Vol. 3, Teil 3Ab12, pp. 1-139, 1922.

Received January 7, 1999, and in final form July 16, 1999. Online publication May 3, 2000. 